Pacific Journal of Mathematic 


\section{FINITE GROUPS WITH A STANDARD COMPONENT OF TYPE $J_{4}$}

\section{LARRY FINKELSTEIN}

In this paper, it is shown that if $G$ is a core-free group with a standard component $A$ of type $J_{4}$, then either $A$ is normal in $G$ or the normal closure of $A$ in $G$ is isomorphic to the direct product of two copies of $J_{4}$.

1. Introduction. Janko [17] has recently given evidence for the existence of a new finite simple group. In particular, Janko assumes that $G$ is a finite simple group which contains an involution $z$ such that $H=C(z)$ satisfies the following conditions:

(i) The subgroup $E=O_{2}(H)$ is an extra-special group of order $2^{13}$ and $C_{H}(E) \leqq E$.

(ii) $H$ has a subgroup $H_{0}$ of index 2 such that $H_{0} / E$ is isomorphic to the triple cover of $M_{22}$.

He then shows that $G$ has order $2^{21} \cdot 3^{3} \cdot 5 \cdot 7 \cdot 11^{3} \cdot 23 \cdot 39 \cdot 31 \cdot 37 \cdot 43$ and describes the conjugacy classes and subgroup structure of $G$. In this paper we shall assume that $J_{4}$ is a finite simple group which satisfies Janko's assumptions and shall prove

THEOREM A. Let $G$ be finite group with $O(G)=1$, A a standard component of $G$ isomorphic to $J_{4}$ and $X=\left\langle A^{G}\right\rangle$. Then either $X=A$ or $X \cong A \times A$.

Our proof follows the outline given in [6] and makes use of two key facts; namely, that $J_{4}$ has a 2-local subgroup isomorphic to the split extension of $E_{2^{11}}$ by $M_{24}$ and that $J_{4}$ has one class of elements of order 3 with the centralizer of an element of order 3 isomorphic to the full cover of $M_{22}$. We also make use of the characterization of finite groups with a standard component isomorphic to $M_{24}$ which was recently obtained by Koch [18].

2. Properties of $J_{4}$. In this section, we shall describe certain properties of $J_{4}$ and its subgroups which will be required for the proof of Theorem A. Most of these properties are found in [17] and will be listed without proof. $A$ will denote a group isomorphic to $J_{4}$.

(2.1) $A$ has 2 classes of elements of order 2 denoted by $\left(2_{1}\right)$ and (2). If $t \in\left(2_{1}\right)$ and $E=O_{2}(C(t))$, then $E$ is isomorphic to an extra special group of order $2^{13}, C(E)=Z(E), O_{2,3}(C(t)) / E$ has order 3 and 
$C(t) / O_{2,3}(C(t)) \cong \operatorname{Aut}\left(M_{22}\right)$. Moreover, if $\langle\beta\rangle \in \operatorname{Syl}_{3}\left(O_{2,3}(C(t))\right)$, then $\langle\beta\rangle$ acts regularly on $E / Z(E)$. For $x \in\left(2_{2}\right), C(x)$ is isomorphic to a split extension of $E_{2^{11}}$ by Aut $\left(M_{22}\right)$ with $C(x)$ acting indecomposably on $\mathrm{O}_{2}(C(x))$.

(2.2) $A$ has one class of elements of order 3. If $\gamma \in A$ has order 3 , then $C(\gamma)$ is isomorphic to the 6-fold cover of $M_{22}$.

(2.3) $A$ has two classes of elements of order 7. If $\delta \in A$ has order 7 , then $C_{4}(\delta) \cong Z_{7} \times S_{5}$ and $\delta \not \delta^{-1}$.

(2.4) Let $T_{0} \in \operatorname{Syl}_{2}(A)$. Then $T_{0}$ has precisely one $E_{2^{11}}$ subgroup, denoted by $U$. $N(U)=U K$ where $K \cong M_{24}$. The orbits of $K$ on $U^{\sharp}$ are $\left(2_{1}\right) \cap U$ of order $7 \cdot 11 \cdot 23$ and $\left(2_{2}\right) \cap U$ of order $4 \cdot 3 \cdot 23$.

In the above, $U$ is isomorphic to the so-called "Fischer" module for $M_{24}$. The following is an important property of the Fischer module.

(2.5) Let $\left(^{*}\right) 1 \rightarrow R \rightarrow V \rightarrow U \rightarrow 1$ be an extension of $F_{2} M_{24}$ modules where $R$ is a trivial module of dimension 1 and $U$ is isomorphic to the Fischer module. Then the extension splits.

Proof. Let $\tilde{U}$ and $\tilde{V}$ be the $F_{2} M_{24}$ modules dual to $U$ and $V$ respectively. Then we have the extension ( $\tilde{*}) 1 \rightarrow \widetilde{U} \rightarrow \widetilde{V} \rightarrow R \rightarrow 1$. It suffices to show that $(\tilde{*})$ splits. Since $U$ is not a self dual module and since there exists precisely 2 nonisomorphic $F_{2} M_{24}$ modules of dimension 11 (see James [16]), $\widetilde{U}$ is isomorphic to the so-called Conway module [5]. Thus $M_{24}$ has 2 orbits on $(\widetilde{U})^{\sharp}$. If $u_{1}$ and $u_{2}$ are representatives of these 2 orbits, then $C_{M_{24}}\left(u_{1}\right) \cong \mathrm{Hol}\left(E_{16}\right)$ and $C_{M_{24}}\left(u_{2}\right) \cong \operatorname{Aut}\left(M_{12}\right)$.

Since $|\widetilde{V}|=2^{12}$, there exists a vector $v \in \widetilde{V}-\widetilde{U}$ such that $v$ is fixed by a Sylow 23 subgroup $S$ of $M_{24}$. The orbit of $M_{24}$ on $(\tilde{V})^{\sharp}$ which contains $v$ has order $\left[M_{24}: C_{M_{24}}(v)\right]$ and is not divisible by 23 . Therefore, by examining the list of maximal subgroups of $M_{24}$ [5], together with $\left[M_{24}: C_{M_{24}}(v)\right] \leqq 2^{12}$, we see that $C_{M_{24}}(v)$ contains a subgroup $L$ isomorphic to $M_{23}$. Consider the action on $\tilde{V}$ of an $M_{22}$ subgroup $M$ of $L$. Then $M$ has no fixed points on $\widetilde{U}^{\sharp}$, so in fact $C_{\breve{V}}(M)=\langle v\rangle$. Therefore $N_{M_{24}}(M) \cong \operatorname{Aut}\left(M_{22}\right)$ fixes $\langle v\rangle$ as well. Finally $\left\langle L, N_{M_{24}}(M)\right\rangle=M_{24}$ centralizes $\langle v\rangle$ and the extension splits.

We shall denote by $E_{2^{11}} \cdot M_{24}$ a split extension of $E_{2^{11}}$ by $M_{24}$ in which $E_{2^{11}}$ is $F_{2} M_{24}$ isomorphic to the Fischer module.

(2.6) Let $M=U K$ be isomorphic to $E_{2^{11}} \cdot M_{24}$ with $U=O_{2}(M)$ 
and $K \cong M_{24}$. Then the classes of elements of order 2 and 3 of $M$ and the orders of the centralizers in $M$ of $a$ representative $\lambda$ are as follows

\begin{tabular}{ccl} 
Class & $\left|C_{U}(\lambda)\right|$ & $\left|C_{M}(\lambda)\right|$ \\
\hline$\left(2_{1}\right)$ & $2^{11}$ & $2^{21} \cdot 3^{3} \cdot 5$ \\
$\left(2_{2}\right)$ & $2^{11}$ & $2^{19} \cdot 3^{2} \cdot 5 \cdot 7 \cdot 11$ \\
$\left(2_{3}\right)$ & $2^{7}$ & $2^{17} \cdot 3 \cdot 7$ \\
$\left(2_{4}\right)$ & $2^{7}$ & $2^{17} \cdot 3$ \\
$\left(2_{5}\right)$ & $2^{6}$ & $2^{15} \cdot 3 \cdot 5$ \\
$\left(2_{6}\right)$ & $2^{6}$ & $2^{15} \cdot 3 \cdot 5$ \\
$\left(3_{1}\right)$ & $2^{5}$ & $2^{8} \cdot 3^{3} \cdot 5$ \\
$\left(3_{2}\right)$ & $2^{3}$ & $2^{6} \cdot 3^{2} \cdot 7$
\end{tabular}

Moreover, if $\lambda_{i} \in\left(3_{i}\right) \cap K$ then $C_{M}\left(\lambda_{i}\right)=C_{U}\left(\lambda_{i}\right) C_{K}\left(\lambda_{i}\right)$ with $C_{K}\left(\lambda_{1}\right)$ isomorphic to the 3 -fold cover of $A_{6}, C_{K}\left(\lambda_{2}\right) \cong Z_{3} \times L_{2}(7)$ and where $C_{K}\left(\lambda_{i}\right) /\left\langle\lambda_{i}\right\rangle$ acts faithfully on $C_{U}\left(\lambda_{i}\right), i=1,2$.

Proof. Let $\lambda$ be an involution of $M-U, \mathcal{O}_{1}, \mathcal{O}_{2}, \cdots, \mathcal{O}_{n}$ the orbits of $C_{M}(\lambda U / U)$ on $\lambda C_{U}(\lambda)$ and $\alpha_{i}$ an element of $\mathscr{O}_{i}, i=1, \cdots, n$. Then $\alpha_{i}$ is conjugate to $\alpha_{j}$ in $M$ exactly when $i=j$ and also $\left|C_{M}\left(\alpha_{i}\right)\right|=\left|C_{M}(\lambda U)\right| /\left|\mathcal{O}_{i}\right|$. Now $K$ has 2 classes of involutions with representatives $\lambda$ and $\eta$ having centralizers in $K$ of order $2^{10} \cdot 3 \cdot 7$ and $2^{9} \cdot 3 \cdot 5$ respectively. Noting that the action of $K$ on $U$ is dual to the action of $K$ on the Conway module, it is easy to see that $\left|C_{U}(\lambda)\right|=2^{7}$ and $\left|C_{U}(\eta)\right|=2^{6}$. Observe that $U$ has 8 orbits on $\lambda C_{U}(\lambda)$, each of which has length 16 . Moreover an element of order 7 of $C_{K}(\lambda)$ fixes 2 points of $C_{U}(\lambda)$ and therefore must permute 7 of these orbits. Since $\left|C_{M}(\lambda)\right|=\left|C_{K}(\lambda)\right|\left|C_{U}(\lambda)\right|=2^{17} \cdot 3 \cdot 7$, it then follows that $C_{M}(\lambda U / U)$ acting on $\lambda C_{U}(\lambda)$ has one orbit of length 16 and one orbit of length $7 \cdot 16=112$ with $\lambda$ an element of the orbit of length 16 . This accounts for the classes $\left(2_{3}\right)$ and $\left(2_{4}\right)$. Similar reasoning accounts for the classes $\left(2_{5}\right)$ and $\left(2_{6}\right)$. We already know from (2.4) that $M$ has orbits on $U^{\#}$ of lengths $4 \cdot 3 \cdot 23$ and $7 \cdot 11.23$ and thus the classes of involutions of $M$ are as described.

Let $\gamma$ and $\tau$ be representatives of the classes of element of order 3 of $K$ with $C_{K}(\gamma)$ isomorphic to the 3-fold cover of $A_{6}$ and $C_{K}(\tau) \cong Z_{3} \times L_{2}(7)$. Clearly $\gamma$ and $\tau$ are representatives of the 2 classes of elements of order 3 of $M$. It suffices to determine the orders of $C_{U}(\gamma)$ and $C_{U}(\tau)$. As before, we may appeal to the action of $K$ on the Conway module to obtain $\left|C_{U}(\gamma)\right|=2^{5}$ and $\left|C_{U}(\tau)\right|=2^{3}$ as required.

Notation. If $H$ is a simple group, then $n H$ will denote a proper 
$n$-fold covering of $H$. If the multiplier of $H$ is cyclic, then $n H$ is unique up to isomorphism. Also let $E_{32} \cdot 3 A_{6}$ be the group isomorphic to the centralizer of an element of order 3 of the class $\left(3_{1}\right)$ of $E_{2^{11}} \cdot M_{24}$. Note that $E_{32} \cdot 3 A_{6}$ is isomorphic to a 2-local subgroup of $6 M_{22}$.

(2.7) The Schur multiplier of $J_{4}$ is trivial.

Proof. See Griess [14].

(2.8) $\operatorname{Aut}\left(J_{4}\right) \cong J_{4}$.

Proof. Let $A \cong J_{4}$ and suppose that $\alpha \in$ Aut $(A)$. We may imbed $A$ in $A$ ut $(A)$ and assume by way of a contradiction that $\alpha \notin A$ but $\alpha^{p} \in A$ for some prime $p$. Set $G=\langle A, \alpha\rangle$.

By (2.4), we may assume that $\alpha \in N_{G}(U)$ where $U$ is an $E_{2^{11}}$ subgroup of $A, N_{A}(U)=U K \cong E_{2^{11}} \cdot M_{24}$ and $K \cong M_{24}$. Since Aut $(K) \cong$ $K$, we may further assume that $\overline{N_{G}(U)}=N_{G}(U) / U=\langle\bar{\alpha}\rangle \times \bar{K}$. It is known [16] that $U$ is an absolutely irreducible $F_{2} K$ module, hence by a result of Schur, we have $[\alpha, U]=1$. Two cases now arise; namely $[\alpha, K]=1$ and $[\alpha, K] \neq 1$.

If $[\alpha, K] \neq 1$, then it is clear that $\alpha$ is a 2-element. Also the fact that $\sigma^{1}(\langle U, \alpha\rangle)$ is a proper $K$ invariant subgroup of $U$ forces $\sigma^{1}(\langle U, \alpha\rangle)=1$. Hence $\langle U, \alpha\rangle \cong E_{2^{12}}$ and $K$ acts indecomposably on $\langle U, \alpha\rangle$. Without loss, we may assume that $\alpha$ is centralized by a Sylow 23 subgroup of $K$. By arguing as in (2.5), it then follows that $C_{K}(\alpha) \cong M_{23}$. Therefore in either case, we have that $C_{U K}(\alpha) \geqq$ $U K_{0}$ where $K_{0}$ is an $M_{23}$ subgroup of $K$.

Let $\gamma$ be an element of order 3 of $K_{0}$. Then $C_{K_{0}}(\gamma) \cong Z_{3} \times A_{5}$ implies that $C_{U}(\gamma) \cong E_{32}$ by (2.6). Also $C_{A}(\gamma) \cong 6 M_{22}$ and $m_{2}\left(C_{A}(\gamma)\right)=5$ [4] gives $O_{2}\left(C_{A}(\gamma)\right) \leqq C_{U}(\gamma)$. Setting $\overline{C_{A}(\gamma)}=C_{A}(\gamma) / Z\left(C_{A}(\gamma)\right) \cong M_{22}$, we conclude that $\alpha$ centralizes a subgroup of $\overline{C_{A}(\gamma)}$ isomorphic to a split extension of $E_{16}$ by $A_{5}$. But no nontrivial automorphism of $M_{22}$ centralizes such a subgroup [9] and therefore $\left[\alpha, C_{A}(\gamma)\right] \leqq Z\left(C_{A}(\gamma)\right)$. $\mathrm{Bv}$ the 3-subgroup lemma, we then have $C_{A}(\gamma) \leqq C_{A}(\alpha)$. Since $\gamma$ is inverted by an element of $K_{0} \leqq C_{A}(\alpha)$, it follows that $N_{A}(\langle\gamma\rangle) \leqq C_{A}(\alpha)$ as well.

Finally, let $\langle t\rangle=O_{2}\left(C_{A}(\gamma)\right)$ so that $C_{A}(t)=E \cdot N_{A}(\langle\gamma\rangle)$ by (2.1), where $E=O_{2}\left(C_{A}(t)\right)$ is extra special of order $2^{13}$. Observe that $C_{A}(\gamma)$ acts irreducibly on $E /\langle t\rangle$. Combining this with $\left[C_{A}(\gamma), \alpha\right]=1$ and $\left.C_{E}(\alpha) \geqq U \cap E\right\rangle\langle t\rangle$, we conclude that $E \leqq C_{A}(\alpha)$. Therefore we are in the position where $C_{A}(\alpha) \geqq C_{A}(t)$ and $C_{U K}(\alpha)=U K_{0}$ or $U K$ with $K_{0} \cong M_{23}$. But $C_{A}(t)$ contains a Sylow 2 subgroup of $N_{A}(U)$ implies that $C_{U K}(\alpha)=U K$ and this gives $C_{A}(\alpha) \geqq\left\langle U K, C_{A}(t)\right\rangle$. An easy argu- 
ment shows that $C_{A}(\alpha)$ is simple with $C_{C_{A}(\alpha)}(t)=C_{A}(t)$. Thus by Janko's theorem [17], $\left|C_{A}(\alpha)\right|=|A|$ which of course gives $A=C_{A}(\alpha)$, a contradiction.

3. Preliminary results. In this section we present certain technical results which are necessary for the proof of Theorem A.

(3.1) Let $G$ be a group, $A$ a standard component of $G$ with $C(A)$ of 2 rank 1. Let $S \in \operatorname{Syl}_{2}(N(A))$. Assume that $S \notin \operatorname{Syl}_{2}(G)$ and $Z(S) \leqq A C(A)$. Then $[A, O(G)]=1$.

\section{Proof. See Seitz [19].}

(3.2) Let $M$ be a group containing an involution $z$ such that $C(z)=O(C(z)) \times\langle z\rangle \times U K$ where $K \cong M_{24}$ and $U$ is $F_{2} K$ isomorphic to the Fischer module. Let $V=\langle z, U\rangle$ and $N=N(V)$. Then either

(i) $z \in Z(N)$ or

(ii) $N=O(N) \times W K$ where $W=\langle z\rangle Y$ is special of order $2^{23}$ with $Z(W)=U$ and where $Y$ is a homocyclic abelian group of order $2^{22}$ invariant under $K$ with $Y / U F_{2} K$ isomorphic to $U$.

Proof. Assume that $z \notin Z(N)$ and let $\bar{N}=N / O(N)$. By (2.2), the orbits of $K$ on $U^{\sharp}$ are $t^{K}$ of order 1771 and $x^{K}$ of order 276 with $C_{K}(x) \cong \operatorname{Aut}\left(M_{22}\right)$. Moreover both $t$ and $x$ are squares in $U K$, hence $z^{N} \cap U=\varnothing$. Now the orbits of $C(z)$ on $V^{\#}$ are precisely

\begin{tabular}{lccccc} 
Orbit & $\{z\}$ & $t^{K}$ & $x^{K}$ & $(z t)^{K}$ & $(z x)^{K}$ \\
\hline Length & 1 & 1771 & 276 & 1771 & 276
\end{tabular}

Since $z \notin Z(N)$ and $z^{N} \cap U=\varnothing, z^{N}$ must be a union of some of the sets $\{z\},(z t)^{K},(z x)^{K}$. But $\left|z^{N}\right|$ is a divisor of $\left|L_{12}(2)\right|$ then gives $z^{N}=z U$.

Representing $N$ on $z^{N}=z U$, we have $|N|=2^{11}\left|N_{H}(V)\right|$, hence $|\bar{N}|=2^{23}\left|M_{24}\right|$. Moreover $U$ is generated by those involutions of $V$ not conjugate to $z$ so that $U \triangleleft N$. Assume that $C_{N}(U)=O(N) V$. Then $\bar{N} / \bar{V}$ acts faithfully on $\bar{U}$ and is therefore isomorphic to a subgroup of $L_{11}(2)$. Let $S \in \operatorname{Syl}_{11}(K)$ so that $N_{K}(S)$ is isomorphic to a Frobenius group of order 10.11. Since $S$ fixes 2 points of $z N$, it follows that $\left|C_{\bar{N}}(\bar{S})\right|=2\left|C_{\bar{N}}(\langle\bar{z}, \bar{S}\rangle)\right|=2^{3} \cdot 11$. Hence a Sylow 11 subgroup of $\bar{N} / \bar{V}$ has centralizer of even order which contradicts the fact that a Sylow 11 subgroup of $L_{11}(2)$ has centralizer of odd order. We conclude that $C_{N}(U)$ properly contains $O(N) V$.

It is easy to see from the action of $K$ on $\overline{C_{N}(U)}$ that $C_{N}(U)=$ $O(N) W$ where $W / U \cong E_{2^{12}}$. Furthermore, $C_{W}(z)=V$ implies that $Z(W)=U$ and $[z, W]=U$. Thus $W$ is a special 2-group of order 
$2^{23}$ with $Z(W)=U$. We will in fact show that $N=O(N) \times W K$. To see this, observe that $V\left\langle K^{N}\right\rangle$ covers $\bar{N}$ together with $[V K, O(N)]=$ 1 implies that $N=O(N) C_{N}(O(N))$. A simple argument establishes that $O^{2^{\prime}}\left(C_{N}(O(N))\right)=W K$ and therefore $N=O(N) \times W K$. For the remainder of the proof, we may assume that $O(N)=1$.

Consider the homomorphism $\varphi: W \rightarrow U$ by $\varphi(w)=[z, w]$. It is easy to see that $\varphi$ induces an $F_{2} K$ isomorphism between $W / V$ and $U$. But then $W / U$ is an $F_{2} K$ module which satisfies, the hypotheses of (2.5) and thus $W / U=V / U \times Y / U$ where $Y / U$ is $F_{2} K$ isomorphic to $U$. It remains for us to show that $Y$ is a homocyclic abelian group. Assume not. Then by the action of $K$ on $Y, Z(Y)=U$. Let $L$ be a subgroup of $K$ isomorphic to Aut $\left(M_{22}\right)$. It follows from the properties of the Fischer module that $\left|C_{Y / U}(L)\right|=\left|C_{U}(L)\right|=2$ with $C_{Y / U}(L)$ and $C_{U}(L)$ the unique proper $L$ invariant submodules of $Y / U$ and $U$ respectively. Let $\langle y U\rangle=C_{Y / U}(L)$ so that $L$ normalizes $\langle y, U\rangle$. Since $y \notin Z(Y), \quad 1 \neq[y, Y]<U$ and since $L$ normalizes $[\langle y, U\rangle, Y]=[y, Y]$ we must have $[y, Y]=C_{U}(L)$. This in turn implies that $\left[Y: C_{Y}(y)\right]=2$. But $L$ normalizes $C_{Y}(\langle y, U\rangle)=C_{Y}(y)$, hence $C_{Y}(y) / U$ as well and this gives a contradiction.

(3.3) Let $Y \cong E_{2^{22}}$ and $M$ a subgroup of Aut( $\left.Y\right)$ such that $M=M_{1} \times M_{2}$ with $M_{1} \cong M_{2} \cong M_{24}$. Then $Y=Y_{1} \oplus Y_{2}$ where $\left[Y_{\imath}, M_{i}\right]=$ $Y_{i}$ and $\left[Y_{i}, M_{j}\right]=0, i \neq j$.

Proof. Let $\gamma$ be an element of order 23 of Aut $(Y)$. If $\gamma$ acts regularly on $Y$, then $C_{\mathrm{Aut}(Y)}(\gamma)$ is isomorphic to $G L_{2}\left(2^{11}\right)$ or is cyclic. Otherwise $\operatorname{dim}\left(C_{Y}(\gamma)\right)=11$ and $C_{\mathrm{Aut}(Y)}(\gamma) \cong Z_{1023} \times L_{11}(2)$. Let $\gamma_{i} \in M_{i}$ be an element of order 23. Then it is clear that $\operatorname{dim}\left(C_{Y}\left(\gamma_{i}\right)\right)=11$. If we set $Y_{i}=C_{Y}\left(\gamma_{j}\right), i \neq j$, then an easy argument verifies that $Y_{1}$ and $Y_{2}$ satisfy $\left[Y_{i}, M_{j}\right]=0, i \neq j$ and $\left[Y_{i}, M_{i}\right]=Y_{i}, i=1,2$ as required.

In the next result, we list certain properties of $2 M_{22}$ which are required for (3.5).

(3.4) Let $D \cong 2 M_{22}, T \in \operatorname{Syl}_{2}(D)$. Then

(i) $D$ has 3 classes of involutions.

(ii) $Z(T)$ has order 4 and contains representatives of the classes of involutions of $D$.

(iii) $T$ has precisely $2 E_{32}$ subgroups, say $F_{1}$ and $F_{2}$. Each is normal in $T$ and self-centralizing in $D$. Also $N\left(F_{1}\right) / F_{1} \cong A_{6}$ and $N\left(F_{2}\right) / F_{2} \cong S_{5}$.

Proof. See Burgoyne and Fong [4].

(3.5) Let $\Gamma$ be a group with an involution $z$ such that $C(z)=$ 
$O(C(z)) D\langle z\rangle$ with $D=E(C(z))$ and $D / O(D) \cong 2 M_{22}$. Assume further that $\Gamma$ has a 2-subgroup $R^{*}=\left(R_{1} \times R_{2}\right)\langle z\rangle$ where $R_{2}=R_{1}^{z}$ has type $2 M_{22}$ and $R=R_{1} \times R_{2} \leqq O^{2}(\Gamma)$. Then $\Gamma=O(\Gamma) E(\Gamma)\langle z\rangle$ with $E(\Gamma) / O(E(\Gamma)) \cong$ $2 M_{22} \times 2 M_{22}$.

Proof. By assumption and (3.4)(iii), $R$ has a normal subgroup $V=V_{1} \times V_{2}$ where $V_{i} \triangleleft R_{i}$ and $V_{i} \cong E_{32}, i=1$, 2. If $\alpha$ is an involution of $R$, then $m_{2}\left(C_{V_{i}}(\alpha)\right) \geqq 3, \quad i=1,2$, gives $m_{2}\left(C_{R}(\alpha)\right) \geqq 7$. Since $m_{2}(C(z))=6$, it follows that $z^{T} \cap R=\varnothing$. Also all involutions of $R^{*}-R$ are conjugate to $z$ which then implies that $z^{\Gamma} \cap R^{*}=z^{R^{*}}$. Since $C_{R^{*}}(z) \in \operatorname{Syl}_{2}(C(z))$, we see that $R^{*} \in \operatorname{Syl}_{2}(\Gamma)$. Furthermore by the Thompson transfer lemma and assumption, $z \notin O^{2}(\Gamma)$ and $R \in$ $\operatorname{Syl}_{2}\left(O^{2}(\Gamma)\right)$. Let $\Lambda=O^{2}(\Gamma)$.

We now examine the structure of $C(D)$. Observe that $C_{C(D)}(z)=$ $O(C(z))\langle z, t\rangle$ where $\langle t\rangle=O_{2}(D)$. By a result of Suzuki, $C(D)$ has dihedral or semidihedral Sylow 2 subgroups. Let $Z \in \operatorname{Syl}_{2}\left(C_{A}(D)\right)$ so that $\langle Z, z\rangle \in \operatorname{Syl}_{2}(C(D))$. Since $C_{R}(z) \in \operatorname{Syl}_{2}(D)$ and $Z(R)=C_{R}\left(C_{R}(z)\right) \in$ $\operatorname{Syl}_{2}\left(C_{A}\left(C_{R}(z)\right)\right)$, we may assume that $Z \leqq Z(R)$. Therefore $Z$ is elementary abelian by (3.4)(ii) and we have either $\langle Z, z\rangle \cong D_{8}$ and $Z \cong E_{4}$, or $Z=\langle t\rangle$. Let $N=N(Z)$ and $\bar{N}=N / Z$. In either case, $\langle\bar{z}\rangle \in \operatorname{Syl}_{2}\left(C_{\bar{N}}(\bar{D})\right)$ and $C_{\bar{N}}(\bar{z}) \leqq N_{\bar{N}}(\bar{D})$ together imply that $\bar{D}$ is a standard component of $\bar{N}$. By Theorem A [8] and (3.1), $E(\bar{N})=$ $\left\langle\bar{D}^{\bar{N}}\right\rangle, Z(E(\bar{N}))$ has odd order and $E(\bar{N}) / Z(E(\bar{N})) \cong M_{22} \times M_{22}$. Let $K=E(N)$ have components $K_{1}$ and $K_{2}$ with $K_{1}^{z}=K_{2}$ and $K_{1} / Z\left(K_{1}\right) \cong$ $M_{22}$. Then $D=C_{K}(D)$ and $D / O(D) \cong 2 M_{22}$ implies that $K / O(K) \cong$ $2 M_{22} \times 2 M_{22}$. Thus $|Z|=4$ and $K=O^{2^{\prime}}\left(C_{A}(Z)\right)$.

Note that $R \leqq K$. Without loss, we may assume that $R_{i} \leqq K_{i}$, $i=1$, 2. By (3.4iii), let $V_{i}$ and $W_{i}$ be the $2 E_{32}$ subgroups of $R_{i}$ with $C_{K_{i}}\left(V_{i}\right)=O\left(K_{i}\right) V_{i}, C_{K_{i}}\left(W_{i}\right)=O\left(K_{i}\right) W_{i}, N_{K_{i}}\left(V_{i}\right) / C_{K_{i}}\left(V_{i}\right) \cong S_{5}$ and $N_{K_{i}}\left(W_{i}\right) / C_{K_{i}}\left(W_{i}\right) \cong A_{6}, i=1$, 2. Set $W=W_{1} \times W_{2}, M=N(W)$ and $\bar{M}=$ $M / W$. Then $\overline{M \cap K}=E(\overline{M \cap K}) O(\overline{M \cap K})$ with $E(\overline{M \cap K}) / O(E(\overline{M \cap K})) \cong$ $A_{6} \times A_{6}$. Since $W_{1}^{z}=W_{2}, C_{M}(z W)=N(\langle z, W\rangle)=W C_{M}(z)$. Also $K=$ $K_{1} K_{2}$ with $K_{1}^{z}=K_{2}$ implies that $C_{M \cap K}(z)$ involves $A_{6}$. Hence by (3.4iii), $C_{\bar{M}}(\bar{z})=\langle\bar{z}\rangle \times O\left(C_{\bar{M}}(\bar{z})\right)(\overline{D \cap M})$ where $\overline{D \cap M}=E\left(C_{\bar{M}}(\bar{z})\right)$ and $\overline{D \cap M} / O(\overline{D \cap M}) \cong A_{6}$. It now follows that $\overline{D \cap M}$ is a standard component of $\bar{M}$ and we have from Proposition 2.3 [7] and (3.1) that $\bar{M}=O(\bar{M}) E(\bar{M})\langle\bar{z}\rangle$ with $E(\bar{M}) / O(E(\bar{M})) \cong A_{6} \times A_{6}$. Furthermore $E\left(\overline{M \cap K)}=E(\bar{M})\right.$ then implies that $Z=C_{W}(E(\bar{M}))$ and this yields $Z \triangleleft M$.

Our next goal is to show that $Z O(\Gamma) \triangleleft \Gamma$. Towards this end, observe that $W, W_{1} \times V_{2}, V_{1} \times W_{2}$ and $V_{1} \times V_{2}$ are the only $E_{2^{10}}$ subgroups of $R$ and that $S_{5}$ is not involved in $N_{A}(W)$ whereas $S_{5}$ is involved in $N_{A}\left(W_{1} \times V_{2}\right), N_{A}\left(V_{1} \times W_{2}\right)$ and $N_{A}\left(V_{1} \times V_{2}\right)$. This prevents $W$ from fusing in $\Lambda$ to $W_{1} \times V_{2}, V_{1} \times W_{2}$ or $V_{1} \times V_{2}$ and 
yields $W \triangleleft N_{A}(R)$. Now $Z(R)$ contains representatives of the classes of involutions of $K$ by (3.4i), hence of $\Lambda$ as well. Since $Z \leqq Z(R)$, $Z$ fails to be strongly closed in $R$ with respect to $\Lambda$ only when $Z^{\lambda} \cap Z(R) \nsubseteq Z$ for some $\lambda \in \Lambda$. If in fact this happens, then we may choose $\lambda \in N_{A}(R)$. But $W \triangleleft N_{A}(R)$ implies that $\lambda \in N_{A}(W)$ and $Z \triangleleft N_{\Lambda}(W)$ then gives $Z^{\lambda}=Z$, a contradiction. Applying Goldschmidt's theorem [11], we conclude that $Z O(\Gamma) \triangleleft \Gamma$. This in turn yields $\Gamma=$ $O(\Gamma) N$.

Since $K=E(N)=O^{2^{\prime}}(N)$, it suffices to show that $[K, O(\Gamma)]=1$. Recall that $E(C(z))=D=C_{K}(z)$. Let $T=C_{R}(z) \in \operatorname{Syl}_{2}(D)$ and $Z(T)=$ $\left\langle t, t_{1}\right\rangle=Z(T) \leqq Z(R)$. Then for $X=O(\Gamma)$, we have $X=C_{X}(z) C_{X}\left(z t_{1}\right) C_{X}\left(t_{1}\right)$. Now $C_{X}(z) \leqq O(C(z))$ and $[O(C(z)), D]=1$ gives $C_{X}(z) \leqq C_{X}\left(t_{1}\right)$. Also $z^{\lambda}=z t_{1}$ for some $\lambda \in Z(R)$, hence $t_{1}=t_{1}^{\lambda} \in D^{\lambda}=E\left(C\left(z t_{1}\right)\right)$. By the same reasoning, $C_{X}\left(z t_{1}\right) \leqq C_{X}\left(t_{1}\right)$ and so $\left[t_{1}, X\right]=1$. But $\left\langle t_{1}^{K}\right\rangle=K$ and therefore $[K, X]=1$ as required.

The next result will be used in conjunction with (3.5).

(3.6) Let $\Gamma_{0}=\Gamma_{1} \times \Gamma_{2}$ with $\Gamma_{1} \cong \Gamma_{2} \cong 6 M_{22}$ and suppose $H=$ $H_{1} \times H_{2}$ is a perfect subgroup of $\Gamma_{0}$. Then by reindexing if necessary $H_{1} \leqq \Gamma_{1}$ and $H_{2} \leqq \Gamma_{2}$.

Proof. Let $\widetilde{\Gamma}_{0}=\Gamma_{0} / \Gamma_{1}$ and observe that $\widetilde{H}=\widetilde{H}_{1} \widetilde{H}_{2}$ where $\widetilde{H}_{i}$ is perfect and $\left[\widetilde{H}_{1}, \widetilde{H}_{2}\right]=1$. Since $\widetilde{\Gamma}_{0} \cong 6 M_{22}$ and $6 M_{22}$ contains no subgroup which is the central product of two proper perfect subgroups (see Conway [5], p. 235), $\widetilde{H} \neq 1$ and either $H_{1} \leqq \Gamma_{1}$ or $H_{2} \leqq \Gamma_{1}$. Assume that $H_{1} \leqq \Gamma_{1}$. Then by the same reasoning applied to $\Gamma_{0} / \Gamma_{2}$, we have $H_{2} \leqq \Gamma_{2}$.

4. Proof of Theorem A. Let $G$ be a group with $O(G)=1$, $A$ a standard component of $G$ with $A / Z(A) \cong J_{4}$ and $X=\left\langle A^{G}\right\rangle$. Furthermore, let $K=C(A)$ and $R \in \operatorname{Syl}_{2}(K)$. It follows from (2.7) that $Z(A)=1$ and from (2.8) that $N(A)=K A$. We shall assume that $G$ is a minimal counterexample to Theorem A. Thus $X \neq A$ whereupon $X$ is simple and $G \leqq \operatorname{Aut}(X)$ by Lemma 2.5 [1].

(4.1) $|R|=2$. Consequently $G=\langle X, R\rangle$.

Proof. Let $g \in G-N(A)$ be chosen so that $Q=K^{g} \cap N(A)$ has a Sylow 2 subgroup $T$ of maximal order. If $m(R)>1$, then by ([3], (3.2) and (3.3)), $R$ is elementary abelian and we may choose $g$ so that $T=R^{g}$. On the other hand, if $m(R)=1$ and $T$ is trivial, then $\Omega_{1}(R)$ is isolated in $C\left(\Omega_{1}(R)\right)$, hence $\Omega_{1}(R)$ is contained in $Z^{*}(G)$ by [10] contradicting $F^{*}(G)$ is simple. Thus in either case, we may assume that $T$ is nontrivial. 
Now $Q=N(A)=K \times A$ implies that $T$ is isomorphic to a subgroup of $A$ under the projection map $\pi: N(A) \rightarrow A$. An easy argument shows that $Q$ is tightly embedded in $Q A$. Moreover, $\pi(Q)^{a}=$ $\pi\left(Q^{a}\right)$ for $a \in A$ then implies that $\pi(Q)$ is normalized by $\left\langle C_{A}(a): a \epsilon\right.$ $\left.\pi(T)^{\sharp}\right\rangle$. Assume first that $m(R)>1$ so that $R$ is elementary abelian and $T=R^{g}$. Let $a \in \pi(T)^{\sharp}$. Then $\pi(Q) \cap C_{A}(\alpha)$ is a normal subgroup of $C_{A}(a)$ with Sylow 2 subgroup $\pi(T) \cong T$. The structure of $C_{A}(a)$ is given in (2.1) and from this we conclude that a belongs to the class $\left(2_{2}\right)$ of $A$ and $\pi(Q) \cap C_{A}(\alpha)=\pi(T) \cong E_{2^{11}}$. But $\pi(T)$ also contains involutions of the class $\left(2_{1}\right)$ and this gives a contradiction.

Assume finally that $m(T)=1$ and let $\langle a\rangle=\Omega_{1}(\pi(T))$. Arguing as before, $\pi(Q) \cap C_{A}(\alpha)$ is a normal subgroup of $C_{A}(\alpha)$ with Sylow 2 subgroup $\pi(T)$, hence by $(2.1), \pi(T)$ has order 2. Since $\pi(T) \cong T$, we may set $T=\langle r a\rangle$ with $1 \neq a \in A$ and $r \in R$. Now $[A, R]=1$ gives $N_{R}(T)=C_{R}(r)$ and since $N_{R}(T) \cong T$ by [2, Theorem 2], we conclude that $R$ has order 2 proving the result.

Since $G$ is a minimal counterexample to Theorem $\mathrm{A}$ and $A$ is a standard component of $\langle R, X\rangle$, with $X=\left\langle A^{X}\right\rangle$, it follows that $\langle R, X\rangle$ is also a counterexample to Theorem $\mathrm{A}$. Hence $G=\langle X, R\rangle$.

Notation. By (4.1), we may set $\langle z\rangle=R$ so that $G=\langle X, z\rangle$. Also $C(z)=O(C(z)) \times\langle z\rangle \times A$ by (2.7) and (2.8). Let $T_{0} \in \operatorname{Syl}_{2}(A)$, $T=\langle z\rangle \times T_{0} \in \operatorname{Syl}_{2}(C(z))$ and $\{V\}=\{\langle z\rangle \times U\}=\mathscr{E}_{12}(T)$ where $U=$ $\mathscr{E}_{11}\left(T_{0}\right)$. Recall from (2.4) that $N_{C(z)}(V)=O(C(z)) \times\langle z\rangle \times U K$ where $U K=N_{A}(U), \quad K \cong M_{24}$ and $U$ is $F_{2} K$ isomorphic to the Fischer module.

$$
\text { (4.2) } z^{G} \cap A=\varnothing \text {. }
$$

Proof. Note that $z$ is not a square in $G$ whereas every involution of $A$ is a square by (2.1).

(4.3) Let $N=N(V)$. Then $z^{G} \cap V=z U . \quad N=O(N) \times W K$ where $W=\langle z\rangle Y$ is special of order $2^{23}$ with $Z(W)=U, Y$ is a homocyclic abelian group of order $2^{22}$ invariant under $K$ and $Y / U$ is $F_{2} K$ isomorphic to $U$.

Proof. Since $C_{N}(z)=O(C(z)) \times\langle z\rangle \times U K$, it suffices, in light of (3.1), to show that $z \notin Z(N)$. Assume in fact that $z \in Z(N)$. Then $V=J(T)$ and $T \in \operatorname{Syl}_{2}(N)$ together imply that $T \in \operatorname{Syl}_{2}(G)$. Furthermore $V$ is weakly closed in $N$ with respect to $G$ and so $N$ controls fusion of $C(V)=O(N) \times V$. But $V$ contains reprssentatives of the classes of involutions of $C(z)$ and therefore $z$ is isolated in $C(z)$. Applying the $Z^{*}$ theorem [10], we then have $z \in Z^{*}(G)$ which is incompatible with $G \leqq \operatorname{Aut}(X)$. 
We continue our analysis using the structure and notation for $N$ set up in (4.3). In order to eliminate the ambiguity in the structure of $Y$ we need the following result.

(4.4) Let $\langle\delta\rangle \in \operatorname{Syl}_{7}(A), \Delta=C(\delta)$ and $\bar{\Delta}=\Delta / O(\Delta)$. Then either $\bar{\Delta} \cong S_{5} 2 Z_{2}$ or $\bar{\Delta}=E(\bar{\Delta})\langle\bar{z}\rangle$ where $E(\bar{A}) \cong U_{3}(5), L_{3}(5)$ or $L_{2}(25)$.

Proof. According to (2.3), $C_{A}(\delta)=\langle\delta\rangle \times D$ where $D \cong S_{5}$. Moreover if $e$ and $d$ are involutions in $D^{\prime}$ and $D-D^{\prime}$ respectively, then by (2.1), $e \in\left(2_{2}\right)$ and $d \in\left(2_{1}\right)$. We shall first show that $z$ fuses to $z d$ and $z e$ in 4 . We know from (4.3) that $z$ fuses to both $z d$ and $z e$ in $G$. Set $H=C(z)$ and assume that $(z d)^{g}=z, g \in G$. Now $C_{H}(z d)^{g}=$ $C(\langle z, z d\rangle)^{g}=C\left(\left\langle z^{g}, z\right\rangle\right)=C_{H}\left(z^{g}\right)$. Since $z^{G} \cap H=\{z\} \cup(z d)^{H} \cup(z e)^{H}$ and $C_{H}(z d) \nRightarrow C_{H}(z e)$, we may replace $g$ by $g h, h \in H$, if necessary, to insure that $z^{g}=z d$. Thus $C_{H}(z d)^{g}=C_{H}(z d)$. Let $B=O^{2^{\prime}}\left(C_{H}(z d)\right)=$ $\langle z\rangle \times C_{A}(d)$ and $B=B / O_{2,3}(B) \cong \operatorname{Aut}\left(M_{22}\right)$. Since $B^{g}=B$ and $\langle\delta\rangle \epsilon$ $\operatorname{Syl}_{7}(B)$, we may assume that $\langle\delta\rangle^{g}=\langle\delta\rangle$. If $\delta^{g} \sim \delta^{-1}$, then $g$ induces an automorphism of $O^{2}(\bar{B}) \cong M_{22}$ in which an element of order 7 is inverted, a contradiction. Therefore $\delta^{g} \sim \delta$ in $U$ and again we may replace $g$ by $g b, b \in B$, if necessary to obtain $\delta^{g}=\delta$ as required. We may prove that $z$ fuses to $z e$ in $\Delta$ in the exact same way making use of the fact that $O^{2^{\prime}}\left(C_{H}(z d)\right) / O_{2}\left(C_{H}(z d)\right) \cong \operatorname{Aut}\left(M_{22}\right)$ by (2.1).

Returning to the structure of $\bar{J}=\Delta / O(\Delta)$, we have $C-(\bar{z})=$ $\overline{O(H}) \times\langle\bar{z}\rangle \times \bar{D}$ so that $\bar{D}^{\prime}$ is standard in $\bar{\Delta}$. Since $\bar{\Delta}$ has sectional 2 rank at most 4 by a result of Harada [14], we may apply the main theorem of [13] to conclude that $E(\bar{J})$ is isomorphic (i) $A_{5}$, (ii) $A_{5} \times A_{5}$, (iii) $L_{3}$ (4), (iv) $M_{12}$, (v) $U_{3}(5)$, (vi) $L_{3}$ (5), (vii) $L_{2}(25)$, or (viii) $A_{7}$. Furthermore except in case (i), $\bar{\Delta} \leqq$ Aut $(E(\bar{\Delta}))$. Since $\overline{z d} \sim \bar{z} \sim \overline{z e}$ in $\bar{d}$, and $\bar{d} \nsim \bar{z} \nsim \bar{e}$ by (4.2), we may easily eliminate cases (i), (iii), (iv) and (viii) and show that in case (ii), $\bar{\Delta} \cong S_{5}<Z_{2}$.

REMARK. If $E(\bar{\Delta})$ is simple then both $O_{2^{\prime}, E}(\Delta)$ and $\Delta-O_{2^{\prime}, E}(\Delta)$ contain one class of involutions. In particular, $z \notin O_{2^{\prime}, E}(\Delta)$ and $d \nsim$ $z \nsim e$ together imply that the classes $\left(2_{1}\right)$ and $\left(2_{2}\right)$ of $A$ fuse in $G$.

\section{(4.5) $\quad Y \cong E_{2^{22}}$.}

Proof. It follows from (4.3) that either the result is true or $Y$ is homocyclic of exponent 4. Assume the latter for purpose of a contradiction. We know that $N=O(N) \times W K$. Thus if $\langle\delta\rangle \epsilon$ $\operatorname{Syl}_{7}(K)$, and $\Delta=C(\delta)$, then the structure of $\bar{\Delta}=\Delta / O(\Delta)$ is given by (4.4). Now $C_{Y}(\delta) \cong Z_{4} \times Z_{4}$ and $C_{K}(\delta)$ contains an element of order 3 which acts regularly on $C_{Y}(\delta)$. This implies that $O^{2}(\Delta)$ contains a $Z_{4} \times Z_{4}$ subgroup and we conclude from (4.4) that $\bar{\Delta}=E(\bar{\Delta})\langle\bar{z}\rangle$ with 
$E(\bar{A}) \cong L_{3}(5)$. Since $E(\bar{A})$ has wreathed Sylow 2 subgroups of order $2^{5}$ and $\bar{z}$ acts as the graph automorphism, $z$ must invert $C_{Y}(\delta)$. But the set of all elements of $Y$ inverted by $z$ forms a subgroup of $Y$ properly containing $U$ and invariant under $K$ which forces $z$ to invert $Y$.

We claim that $Y$ is the unique $\left(Z_{4}\right)^{11}$ subgroup of $N$. In fact let $Y_{1}$ be another such subgroup of $N$. Then $W K=\widetilde{W K} / V \cong E_{2^{11}} \cdot M_{24}$ together with $m_{2}\left(\tilde{Y}_{1}\right)=11$ gives $\widetilde{Y}_{1}=\tilde{W}$. Therefore $Y_{1} \leqq W=\langle z\rangle Y$ and since $z$ inverts $Y$, we must have $Y=Y_{1}$. This in turn implies that $W$ must be the unique subgroup of $N$ of its isomorphism type as well. In particular, if $N=N(W)$, then $W$ is weakly closed in its normalizer with respect to $G$. Hence $N$ contains a Sylow 2 subgroup of $G$ and this in turn forces $N$ to control fusion of $C(W)=$ $O(N) U$. Now the $2 N$ classes of involutions of $U$ are the sets $\left(2_{1}\right) \cap U$ and $\left(2_{2}\right) \cap U$ of $A$. Also in the remark following (4.4), we observed that the classes $\left(2_{1}\right)$ and $\left(2_{2}\right)$ of $A$ fuse in $G$ if $E(\bar{\Delta}) \cong L_{3}(5)$. Thus $N$ must act transitively on $U$ which is clearly not the case and we conclude that $N<N(W)$.

We now investigate the structure of $N(W)$. First observe that $C(W) \leqq C(V)$ gives $C(W)=U O(N)$. Set $\overline{N(W)}=N(W) / U$ and consider the action of $\overline{N(W)}$ on $\bar{W}$. Since $Y$ is characteristic in $W, \bar{Y}$ is normal in $\overline{N(W)}$. Also $C_{\overline{N(W)}}(\bar{z})=\bar{N}=\langle\bar{z}\rangle \times O(\bar{N}) \times \bar{Y} \bar{K}$. Therefore we may apply (3.1) to conclude that $N(W)=O(N) \times W^{*} K$ where $W^{*}$ is a 2-group containing $W$ invariant under $K, W=\langle z\rangle Y^{*}$ where $Y^{*}$ contains $Y$ and is invariant under $K$ with $\bar{Y}^{*} / \bar{Y} F_{2} K$ isomorphic to $\bar{Y}$.

But $Y^{*} / Y, Y / U$ and $U$ are all $F_{2} K$ isomorphic, hence $\left|C_{Y}(\delta)\right|=2^{6}$ and this in turn gives $\left|C_{W^{*}}(\delta)\right|=2^{7}$ which contradicts $|\Delta|_{2}=2^{6}$.

(4.6) $W \in \operatorname{Syl}_{2}(C(U))$. Hence $Y \in \operatorname{Syl}_{2}(C(Y))$.

Proof. The second statement follows easily from the first. Now $z^{G} \cap Y=\varnothing$ together with $z^{N}=z U$ by (4.3) gives $\left\langle z^{G} \cap W\right\rangle=V$. Thus $V$ is weakly closed in $W$ with respect to $G$. This implies that $N_{C(U)}(W)=N \cap C(U)=O(N) \times W$ by (4.3), hence $W \in \operatorname{Syl}_{2}(C(U))$ as required.

(4.7) Let $M=N(Y)$ and $\bar{M}=M / Y$. Then

(i) $C_{\bar{M}}(\bar{z})=\bar{N}=\overline{O(N)} \times\langle\bar{z}\rangle \times \bar{K}$.

(ii) $\bar{z} \notin Z^{*}(\bar{M})$.

Proof. Suppose $z^{\alpha} \in z Y, \alpha \in M$. Since $z^{G} \cap W=z^{W}=z U$ by (4.3), $\alpha w$ normalizes $V$, hence $\alpha w \in N$. This in turn implies that $\alpha \in N$ and we see that $\bar{N}=\overline{C_{M}(z)}=O(\bar{N}) \times\langle\bar{z}\rangle \times \bar{K}$, proving (i). 
To prove (ii), let $b$ be an involution of $U K-U$. Since $z$ fuses to $z \alpha$ for any involution $a \in A$ by (4.3), there exists $g \in G$ such that $z^{g}=z b$. By (2.4), we see that $m_{2}(C(z b))=12$ and all $E_{2^{12}}$ subgroups of $C(z b)$ are conjugate. Therefore $\left\langle z b, C_{Y}(z b)\right\rangle=V^{g h}$ for some $h \epsilon$ $C(z b)$. Observe that $C_{Y}(z b)$ is generated by those involutions of $\left\langle z b, C_{Y}(z b)\right\rangle$ which are not conjugate to $z b$. Hence $U^{g h}=C_{Y}(z b)$. Also $W \in \operatorname{Syl}_{2}(C(U))$ by (4.6) implies that $W^{g h} \in \operatorname{Syl}_{2}\left(C\left(C_{Y}(z b)\right)\right)$. Since $\langle Y, z b\rangle \in \operatorname{Syl}_{2}\left(C\left(C_{Y}(z b)\right)\right)$ as well, there exists $k \in G$ such that $W^{g h k}=$ $\langle Y, z b\rangle$. Finally, $z^{g h t} \in z^{G} \cap\langle Y, z b\rangle=(z b)^{Y}$ implies that $z^{g h k l}=z b$ for $l \in\langle Y, z b\rangle$. Setting $g^{\prime}=g h k l$, we have $z^{g^{\prime}}=z b$ and $W^{g}=\langle Y, z b\rangle$. Therefore $Y^{g^{\prime}}=Y$ and $z \sim z b$ in $M$. We have shown that $\bar{z} \sim \overline{z b}$ in $\bar{M}$ and thus $\bar{z} \notin Z^{*}(\bar{M})$.

\section{(4.8) $\quad M=O(M)\left(M_{1} \times M_{2}\right)\langle z\rangle$ where $M_{1}^{z}=M_{2} \cong E_{2^{11}} \cdot M_{24}$.}

Proof. If follows from (4.7) that $C_{\bar{M}}(\bar{z})=\langle\bar{z}\rangle \times \bar{K}$ and $\bar{z} \notin Z^{*}(\bar{K})$. Therefore, by a result of Koch [18] and (3.1), $\bar{M}=O(\bar{M}) E(\bar{M})\langle\bar{z}\rangle$ where $E(\bar{M}) \cong M_{24} \times M_{24}$. Let $M_{1}$ and $M_{2}$ be the minimal normal subgroups of $M$ which map onto the direct factors of $E(\bar{M})$. By (3.2), $Y=U_{1} \times U_{2}$ where $\left[M_{i}, U_{i}\right]=U_{i}$ and $\left[M_{i}, U_{j}\right]=1, i \neq j$. It is clear that either $O_{2}\left(M_{i}\right)=U_{i}$ or $O_{2}\left(M_{i}\right)=Y, i=1,2$. Assume the latter happens and set $\widetilde{M}_{1}=M_{1} / U_{1}$. Since $M_{1}$ is perfect and $U_{2}$ is central in $M_{1}, \widetilde{M}_{1}$ is a perfect central extension of $E_{2^{11}}$ by $M_{24}$. But this contradicts the fact that $M_{24}$ has trivial multiplier [4]. Therefore $O_{2}\left(M_{i}\right)=U_{i}, \quad i=1,2$. Now $M_{1} \cap M_{2} \leqq O_{2}\left(M_{1}\right) \cap O_{2}\left(M_{2}\right)=$ $U_{1} \cap U_{2}=1$ gives $M_{1} M_{2}=M_{1} \times M_{2}$. Finally $M_{1}^{z}=M_{2} \cong C_{M_{1} M_{2}}(z) \cong$ $E_{2^{11}} \cdot M_{24}$ proving the result.

Notation. From (4.8), let $M_{0}=\left(M_{1} \times M_{2}\right)\langle z\rangle$ with $M_{2}=M_{1}^{z} \cong$ $E_{2^{11}} \cdot M_{24}$. Set $M_{1}=U_{1} K_{1}$ with $U_{1}=O_{2}\left(M_{1}\right), K_{1} \cong M_{24}$ and set $M_{2}=$ $U_{2} K_{2}$ with $U_{2}=U_{1}^{z}, K_{2}=K_{1}^{z}$. Furthermore, let $U K=C_{M_{1} M_{2}}(z)$ with $U=C_{U_{1} U_{2}}(z)$ and $K=C_{K_{1} K_{2}}(z)$. Finally, let $S_{1} \in \operatorname{Syl}_{2}\left(M_{1}\right), S_{2}=S_{1}^{z} \epsilon$ $\operatorname{Syl}_{2}\left(M_{2}\right), S=S_{1} \times S_{2}$ and $S^{*}=\langle S, z\rangle \in \operatorname{Syl}_{2}\left(M_{0}\right)$.

(4.9) $S^{*} \in \operatorname{Syl}_{2}(G), S=S^{*} \cap X \in \operatorname{Syl}_{2}(X)$ and $z \notin X$.

Proof. First observe that all involutions of $S^{*}-S$ are conjugate in $S^{*}$ to $z$ and $C_{S^{*}}(z) \in \operatorname{Syl}_{2}(C(z))$. Furthermore, it is easy to see that $z^{G} \cap S=\varnothing$. In fact, if $s$ is an involution of $S$, then $C_{Y}(s)=C_{Y_{1}}(s) \times C_{Y_{2}}(s)$ has order at least $2^{12}$ gives $m_{2}\left(C_{Y}(s)\right) \geqq 13$ whereas $m_{2}(C(z))=12$ by (2.4). Therefore $z^{S^{*}}=z^{G} \cap S$ and we have at once that $S^{*} \in \mathrm{Syl}_{2}(G)$. It is clear from the Thompson transfer lemma that $z \notin O^{2}(G)$. Since $G=\langle X, z\rangle$, we have $X=O^{2}(G)$. Thus $z \notin X$. Also $S \leqq O^{2}\left(M_{0}\right) \leqq X$ gives $S=S^{*} \cap X \in \operatorname{Syl}_{2}(X)$. 
(4.10) Let $\gamma$ be an element of order 3 of $A$ and $\Gamma=C(\gamma)$. Then $\Gamma=O(\Gamma) E(\Gamma)\langle z\rangle$ where $E(\Gamma)=\Gamma_{1} \times \Gamma_{2}$ and $\Gamma_{1}^{z}=\Gamma_{2} \cong 6 M_{22}$.

Proof. First observe from (2.2) that $C_{I}(z)=O(C(z)) \times\langle z\rangle \times C_{A}(\gamma)$ where $C_{A}(\gamma) \cong 6 M_{22}$. Also by (2.2) we may assume that $\gamma$ belongs to the class $\left(3_{1}\right)$ of $U K$. Thus we may write $\gamma=\gamma_{1} \gamma_{2}$ where $\gamma_{2}=\gamma_{1}^{z}$ and $\gamma_{i}$ belongs to the class $\left(3_{1}\right)$ of $M_{i}, i=1,2$. Applying (2.6) gives $C_{M_{0}}(\gamma)=\left(C_{M_{1}}\left(\gamma_{1}\right) \times C_{M_{2}}\left(\gamma_{2}\right)\right)\langle z\rangle$ where $C_{M_{1}}\left(\gamma_{1}\right)^{z}=C_{M_{2}}\left(\gamma_{2}\right) \cong E_{32} \cdot 3 A_{6}$. Since $C_{M_{1}}\left(\gamma_{1}\right)$ is isomorphic to a 2-local subgroup of $6 M_{22}$ which contains a Sylow 2 subgroup of $6 M_{22}$, we may set $R^{*} \in \operatorname{Syl}_{2}\left(C_{M \Sigma_{0}}(\gamma)\right)$ where $R^{*}=$ $\left(R_{1} \times R_{2}\right)\langle z\rangle, R_{2} \in \operatorname{Syl}\left(C_{M_{1}}\left(\gamma_{1}\right)\right)$ and $R_{2}=R_{2}^{z}$ has type $2 M_{22}$. Also $R_{1} \times$ $R_{2} \leqq O^{2}(\Gamma)$. Thus by (3.5), $\Gamma=O(\Gamma) E(\Gamma)\langle z\rangle$ where $E(\Gamma) / O(E(\Gamma)) \cong$ $2 M_{22} \times 2 M_{22}$. But $\left(C_{M_{0}}(\gamma)\right)^{(\infty)}=C_{M_{1}}(\gamma) \times C_{M_{2}}(\gamma) \leqq E(\Gamma)$ then gives $E(\Gamma)=\Gamma_{1} \times \Gamma_{2}$ where $\Gamma_{2}=\Gamma_{1}^{z} \cong 6 M_{22}$.

(4.11) Let $\gamma_{i}$ and $\tau_{i}$ be representatives of the classes $\left(3_{1}\right)$ and $\left(3_{2}\right)$ respectively of $M_{i}$ with $\gamma_{1}^{z}=\gamma_{2}$ and $\tau_{1}^{z}=\tau_{2}$. Let $\gamma=\gamma_{1} \gamma_{2}$ and $\tau=\tau_{1} \tau_{2}$. Then $\gamma_{1} \tau_{2}, \tau_{1} \gamma_{2}, \tau$ and $\gamma$ are conjugate in $X$.

Proof. We know that $\tau$ is conjugate to $\gamma$ in $A$ by (2.2). Since $z$ leaves $\gamma^{X}$ invariant under conjugation and $\left(\tau_{1} \gamma_{2}\right)^{z}=\gamma_{1} \tau_{2}$, it suffices to show that $\tau_{1} \gamma_{2}$ fuses to $\gamma$ in $X$. This in turn may be proved by verifying that $\tau_{1}$ fuses to $\gamma_{1}$ in $C_{X}\left(\gamma_{2}\right)$. Let $P_{i} \in \operatorname{Syl}_{3}\left(M_{i}\right)$ with $P_{1}^{z}=$ $P_{2}, Z\left(P_{i}\right)=\left\langle\gamma_{i}\right\rangle$ and assume that $\tau_{i} \in P_{i}, i=1,2$. Since $C_{M_{0}}(\gamma)^{(\infty)}=$ $C_{M_{1}}\left(\gamma_{1}\right) \times C_{M_{2}}\left(\gamma_{2}\right)$ is contained in $E(\Gamma)=\Gamma_{1} \times \Gamma_{2}$, it follows from (3.6), that subject to reindexing, if necessary, $C_{M_{i}}\left(\gamma_{i}\right) \leqq \Gamma_{i}, i=1,2$. In particular, $P_{i} \in \operatorname{Syl}_{3}\left(\Gamma_{i}\right)$ and $\left\langle\gamma_{i}\right\rangle=O_{3}\left(\Gamma_{i}\right), i=1,2$. Now $P_{1}$ contains an $E_{9}$ subgroup $\left\langle\gamma_{1}, \gamma_{1}^{*}\right\rangle$ all of whose elements of order 3 are conjugate in $M_{1}$ to $\gamma_{1}$. On the other hand, $M_{22}$ contains one class of elements of order 3, hence $\tau_{1}$ is conjugate in $\Gamma_{1}$ to an element of $\left\langle\gamma_{1}, \gamma_{1}^{*}\right\rangle$. Therefore, $\gamma_{1}$ is conjugate to $\tau_{1}$ in $\left\langle M_{1}, \Gamma_{1}\right\rangle \leqq C_{X}\left(\gamma_{2}\right)$ as required.

$$
\text { (4.12) } I\left(S_{i}\right)=U_{i}^{X} \cap I(S) .
$$

Proof. Since $S$ has type $J_{4} \times J_{4}, Y=J(S)$ by (2.4). Therefore $N_{X}(Y)$ controls fusion of $Y$ and we have that $U_{i}^{X} \cap Y=U_{i}, i=1,2$.

We now observe from (2.6) that every involution of $M_{1} M_{2}-Y$ centralizes an element of order 3 of $M_{1} M_{2}$ which is conjugate to $\tau_{1} \tau_{2}=\tau, \gamma_{1} \gamma_{2}=\gamma, \tau_{1} \gamma_{2}$ or $\gamma_{1} \tau_{2}$. Also $C_{M_{i}}\left(\gamma_{i}\right)=C_{U_{i}}\left(\gamma_{i}\right) C_{K_{i}}\left(\gamma_{i}\right) \cong E_{32} \cdot 3 A_{6}$ and $C_{M \mu_{i}}\left(\tau_{i}\right) \cong C_{U_{i}}\left(\tau_{i}\right) C_{K_{i}}\left(\tau_{i}\right) \cong E_{8}\left(L_{3}(2) \times Z_{3}\right)$. In the course of proving (4.11), we showed that up to reindexing, it may be assumed that $C_{M_{i}}\left(\gamma_{i}\right) \leqq \Gamma_{i}, i=1,2$. Let $R=R_{1} \times R_{2} \in \operatorname{Syl}_{2}\left(\Gamma_{1} \Gamma_{2}\right)$ where $R_{i} \in \operatorname{Syl}_{2}\left(\Gamma_{i}\right)$ and $R_{i} \leqq C_{M_{i}}\left(\gamma_{i}\right), i=1,2$. By (3.4), $Z\left(R_{i}\right)$ has order 4 and contains representatives of the 3 classes of involutions of $\Gamma_{i}, i=1,2$. But 
then every involution of $R_{i}$ is conjugate to an element of $Z\left(R_{i}\right)$ whereas every involution of $R-R_{i}$ is conjugate to an element of $Z(R)-Z\left(R_{i}\right)$. Since $Y \cap R=\left(U_{1} \cap R_{1}\right) \times\left(U_{2} \cap R_{2}\right)$ with $U_{i} \cap R_{i} \cong E_{32}$, we have $Z\left(R_{i}\right) \leqq U_{i}$ and $Z(R)-Z\left(R_{i}\right) \leqq U-U_{i}$. Therefore $U_{i}^{X} \cap$ $Y=U_{i}$ then yields $Z\left(R_{i}\right)^{X} \cap Z(R)=Z\left(R_{i}\right)$. We now conclude that $I\left(R_{i}\right)=U_{i}^{X} \cap I(R), i=1,2$ and this in turn gives $I\left(\Gamma_{i}\right)=U_{i}^{X} \cap I(\Gamma)$, $i=1,2$.

Our next objective is to show that $I\left(C_{M_{i}}\left(\tau_{i}\right)\right)=U_{i}^{X} \cap I\left(C_{M_{1} M_{2}}(\tau)\right)$, $i=1$, 2. By (4.11) there exists $g \in X$ such that $\tau^{g}=\gamma$, hence $\left(C_{M_{1} M_{2}}(\gamma)\right)^{g} \leqq C_{X}(\gamma)$. Since $O^{2^{\prime}}\left(C_{M_{1} M_{2}}(\tau)\right)=C_{M_{1}}\left(\tau_{1}\right)^{\prime} \times C_{M_{2}}\left(\tau_{2}\right)^{\prime}$, we have $\left(C_{M_{1}}\left(\tau_{1}\right)^{\prime}\right)^{g} \times\left(C_{M_{2}}\left(\tau_{2}\right)^{\prime}\right)^{g}=O^{2^{\prime}}\left(C_{M_{1} M_{2}}(\tau)\right)^{g} \leqq O^{2 \prime}\left(C_{X}(\gamma)\right)=\Gamma_{1} \Gamma_{2}$ by (3.5). Furthermore by (3.6), $C_{M_{i}}\left(\tau_{i}\right)^{\prime} \leqq \Gamma_{j_{i}}$ with $j_{1} \neq j_{2}$. But $O_{2}\left(C_{M_{i}}\left(\tau_{i}\right)^{\prime}\right)=$ $C_{U_{i}}\left(\tau_{i}\right) \cong E_{8}$ combined with $U_{i}^{X} \cap \Gamma_{i}=I\left(\Gamma_{i}\right)$ yields $\left(C_{M_{i}}\left(\tau_{i}\right)^{\prime}\right)^{g} \leqq \Gamma_{i}$. Therefore $I\left(C_{M_{i}}\left(\tau_{i}\right)^{g}\right)=U_{i}^{X} \cap I\left(C_{M_{1} M_{2}}(\tau)^{g}\right)$ and this implies that $I\left(C_{M_{i}}\left(\tau_{i}\right)\right)=$ $U_{i}^{X} \cap I\left(C_{M_{1} M_{2}}(\tau)\right), i=1,2$. The same argument then gives $I\left(C_{M_{i}}\left(\tau_{i}\right)\right)=$ $U_{i}^{X} \cap I\left(C_{M_{1} M_{2}}\left(\tau_{i} \delta_{j}\right)\right)$ and $I\left(C_{M_{i}}\left(\gamma_{i}\right)\right)=U_{i}^{X} \cap I\left(C_{M_{1} M_{2}}\left(\gamma_{i} \delta_{j}\right)\right), \quad i \neq j, \quad \delta_{j}=\tau_{j}$ or $\gamma_{j}$. Since a conjugate of every involution of $M_{1} M_{2}$ centralizes $\gamma, \tau, \gamma_{1} \tau_{2}$ or $\tau_{1} \gamma_{2}$, we see at once that $I\left(M_{i}\right)=U_{i}^{X} \cap I\left(M_{1} M_{2}\right), i=1,2$. Therefore $I\left(S_{i}\right)=U_{i}^{X} \cap I(S), i=1,2$ proving the result.

\section{(4.13) The following holds:}

(i) $S_{i}$ is a Sylow 2 subgroup of $O^{2}\left(C_{X}\left(S_{j}\right)\right)$ and $O^{2}\left(C_{X}\left(U_{j}\right)\right), i \neq j$.

(ii) Every involution of $S_{i}$ is conjugate in $C_{X}\left(S_{j}\right)$ to an element of $U_{i}, i \neq j$.

Proof. Since $U_{j} \triangleleft S, S_{i} \times U_{j} \in \operatorname{Syl}_{2}\left(C_{X}\left(U_{j}\right)\right), i \neq j$. By Gaschutz's theorem we may write $C_{X}\left(U_{j}\right)=C_{j} U_{j}$ where $C_{j}$ is a complement to $U_{j}$ in $C_{X}\left(U_{j}\right)$. Also $U_{j}$ is central in $C_{X}\left(U_{j}\right)$ gives $C_{X}\left(U_{j}\right)=C_{j} \times U_{j}$. Clearly $O^{2}\left(C_{X}\left(U_{j}\right)\right) \leqq C_{j}$. Also $S_{i} \leqq M_{i}$ and $\left[M_{i}, S_{j}\right]=1$ yields $S_{i} \leqq C_{j}$. It now follows directly that $S_{i} \in \operatorname{Syl}_{2}\left(O^{2}\left(C_{X}\left(U_{j}\right)\right)\right)$. The same proof may be used to verify that $S_{i} \in \operatorname{Syl}_{2}\left(O^{2}\left(C_{X}\left(\mathrm{~S}_{j}\right)\right)\right.$ and this completes the proof of (i).

In order to prove (ii), first observe that $S_{j}=\Omega_{1}\left(S_{j}\right)$, hence by (4.12), $S_{j}$ is weakly closed in $S$ with respect to $X$. Therefore $N_{X}\left(S_{j}\right)$ controls fusion of $C_{X}\left(\mathrm{~S}_{j}\right)$. Since $S_{i} \in \operatorname{Syl}_{2}\left(O^{2}\left(C_{X}\left(S_{j}\right)\right)\right)$ by (i), the Frattini argument gives $N_{X}\left(S_{j}\right)=C_{X}\left(S_{j}\right) N_{X}(S)$. Now $N_{X}(S) \leqq N_{X}(Y)$ where $N_{X}(Y)=M \cap X=O(M)\left(M_{1} \times M_{2}\right)$. Clearly $\bar{S}$ is self normalizing in $\overline{M \cap X}=M \cap X / O(M)$ and this yields $N_{X}(S)=O\left(N_{X}(S)\right) S$. Consequently $N_{X}\left(S_{j}\right)=C_{X}\left(S_{j}\right) S_{j}$. But $\left[S_{i}, S_{j}\right]=1$ implies that $C_{X}\left(S_{j}\right)$ controls fusion of $S_{i} \times Z\left(S_{j}\right) \in \operatorname{Syl}_{2}\left(C_{X}\left(S_{j}\right)\right)$ and the result now follows from (4.12).

(4.14) $S_{i}$ is strongly closed in $S$ with respect to $X, i=1,2$. 
Proof. By symmetry, we need only prove the result for $S_{1}$. Assume in fact that $S_{1}$ is not strongly closed in $S$ with respect to $X$. Let $s_{1} \in S_{1}$ be an element of minimal order of $S_{1}$ such that $s_{1}^{X} \cap$ $S \nsubseteq S_{1}$. Then $s_{1}^{g}=s_{1}^{\prime} s_{2}^{\prime}$ for some $g \in X, s_{i}^{\prime} \in S, \quad \mathrm{i}=1,2$, and $s_{2}^{\prime} \neq 1$. By (4.12), we may assume that $\left|s_{1}\right|>2$. Also $\left(s_{1}^{2}\right)^{g}=\left(s_{1}^{\prime}\right)^{2}\left(s_{2}^{\prime}\right)^{2}$ together with the minimality of $\left|s_{1}\right|$ implies that $s_{2}^{\prime}$ is an involution. By (4.13ii), $s_{2}^{\prime}$ is conjugate in $C_{X}\left(S_{1}\right)$ to an element of $U_{2}$, so we may further assume that $s_{2}^{\prime} \in U_{2}$. But $U_{2}$ is weakly closed in $S$ with respect to $X$ by (2.4) and (4.12), therefore $N_{X}\left(U_{2}\right)$ controls fusion of $C_{X}\left(U_{2}\right)$. A contradiction may now be established by observing that $s_{1} \in S_{1} \in \operatorname{Syl}_{2}\left(O^{2}\left(C_{X}\left(U_{2}\right)\right)\right)$ whereas $s_{1}^{\prime} s_{2}^{\prime} \in O^{2}\left(C_{X}\left(u_{2}\right)\right)$ by (4.13i).

We are now in the position to complete the proof of Theorem A. By (4.14) and the Aschbacher-Goldschmidt theorem [12], $X$ is not simple. This of course contradicts our condition that $X$ is simple and $G \leqq$ Aut $X$.

\section{REFERENCES}

1. M. Aschbacher, Standard components of alternating type centralized by a 4-group, to appear.

2. - Finite groups of component type, Illinois J. Math., 19 (1975), 87-115.

3. M. Aschbacher and G. Seitz, On groups with a standard component of known type, to appear.

4. N. Burgoyne and P. Fong, The Schur multipliers of the Mathieu groups, Nagoya Math. J., 27 (1966), 733-745, Correction 31 (1968), 297-304.

5. J. H. Conway, Three lectures on exceptional groups, article in "Finite Simple Groups," Academic Press, New York, 1971.

6. L. Finkelstein, Finite groups with a standard component isomorphic to $M_{23}$, J. Algebra, 40 (1976), 541-555.

7. - Finite groups with a standard component isomorphic to HJ or HHM, J. Algebra, 43 (1976), 61-114.

8. — Finite groups with a standard component isomorphic to $M_{22}$, J. Algebra, 44 (1977), 558-572.

9. J. S. Frame, Computation of characters of the Higman-Sims group and its automorphism group, J. Algebra, 20 (1972), 320-349.

10. G. Glauberman, Central elements of core-free groups, J. Algebra, 20 (1966), 403420.

11. D. Goldschmidt, 2-Fusion in finite groups, Ann. of Math., 99 (1974), 70-117.

12. - Strongly closed 2-subgroups of finite groups, Ann. of Math., 102 (1975), 475-489.

13. D. Gorenstein and K. Harada, Finite groups whose 2-subgroups are generated by at most 4 elements, Memoir Amer. Math. Soc., No. 147 (1974).

14. R. Griess, Personal communication.

15. K. Harada, On finite groups having self-centralizing 2-subgroups of small order, J. Algebra, 33 (1975), 144-160.

16. G. D. James, The modular characters of the Mathieu groups, J. Algebra, 27 (1973), $57-111$.

17. Z. Janko, A new finite simple group of order $86,775,571,046,077,562,880$, which possesses $M_{24}$ and the full covering group of $M_{22}$ as subgroups, J. Algebra, 42 (1976), 564-596. 
18. J. Koch, Standard components isomorphic to $M_{24}$, unpublished.

19. G. Seitz, Standard subgroups of type $L_{n}\left(2^{a}\right)$, to appear.

Received July 8, 1976 and in revised form January 17, 1977.

Wayne State University

DETROIT, MI 48202 


\section{PACIFIC JOURNAL OF MATHEMATICS}

\section{EDITORS}

RICHARD ARENS (Managing Editor)

University of California

Los Angeles, California 90024

C. W. CURTIS

University of Oregon

Eugene, OR 97403

C. C. MOORE

University of California

Berkeley, CA 94720

\section{J. DugundJI}

Department of Mathematics University of Southern Californıa Los Angeles, California 90007

R. FinN AND J. Milgram Stanford University Stanford, California 94305

\section{ASSOCIATE EDITORS}

E. F. BECKENBACH

B. H. NEUMANN

F. WOLF

K. YOSHIDA

\section{SUPPORTING INSTITUTIONS}

UNIVERSITY OF BRITISH COLUMBIA CALIFORNIA INSTITUTE OF TECHNOLOGY UNIVERSITY OF CALIFORNIA MONTANA STATE UNIVERSITY UNIVERSITY OF NEVADA, RENO NEW MEXICO STATE UNIVERSITY OREGON STATE UNIVERSITY UNIVERSITY OF OREGON OSAKA UNIVERSITY
UNIVERSITY OF SOUTHERN CALIFORNIA STANFORD UNIVERSITY UNIVERSITY OF TOKYO UNIVERSITY OF UTAH WASHINGTON STATE UNIVERSITY UNIVERSITY OF WASHINGTON AMERICAN MATHEMATICAL SOCIETY NAVAL WEAPONS CENTER 


\section{Pacific Journal of Mathematics}

\section{Vol. 71, No. $1 \quad$ November, 1977}

Charalambos D. Aliprantis and Owen Sidney Burkinshaw, On universally complete Riesz spaces ............................. 1

Stephen Richard Bernfeld and Jagdish Chandra, Minimal and maximal solutions of nonlinear boundary value problems .................

John H. E. Cohn, The length of the period of the simple continued fraction of

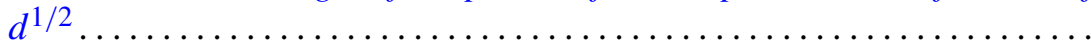

Earl Vern Dudley, Sidon sets associated with a closed subset of a compact abelian group .................................... 33

Larry Finkelstein, Finite groups with a standard component of type $J_{4} \ldots \ldots$

Louise Hay, Alfred Berry Manaster and Joseph Goeffrey Rosenstein, Concerning partial recursive similarity transformations of linearly ordered sets .......................................

Richard Michael Kane, On loop spaces without $p$ torsion. II ............

William A. Kirk and Rainald Schoneberg, Some results on

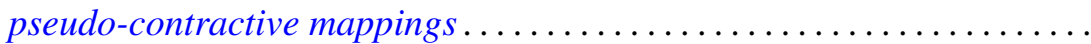

Philip A. Leonard and Kenneth S. Williams, The quadratic and quartic character of certain quadratic units. I. .

Lawrence Carlton Moore, A comparison of the relative uniform topology and the norm topology in a normed Riesz space .................

Mario Petrich, Maximal submonoids of the translational hull 119

Mark Bernard Ramras, Constructing new R-sequences . . .

Dave Riffelmacher, Multiplication alteration and related rigidity properties

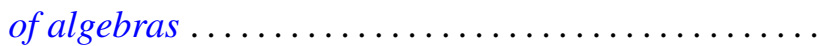

Jan Rosiński and Wojbor Woyczynski, Weakly orthogonally additive functionals, white noise integrals and linear Gaussian stochastic processes.

Ryōtarō Satō, Invariant measures for ergodic semigroups of operators

Peter John Slater and William Yslas Vélez, Permutations of the positive integers with restrictions on the sequence of differences...

Edith Twining Stevenson, Integral representations of algebraic cohomology classes on hypersurfaces ........................

Laif Swanson, Generators of factors of Bernoulli shifts . 